\title{
Review
}

\section{Practice, Progress and Future Directions for Physical Therapies in Huntington's Disease}

\author{
Monica Busse ${ }^{\mathrm{a}, *}$, Hanan Khali1 ${ }^{\mathrm{a}}$, Simon Brooks ${ }^{\mathrm{b}}$, Lori Quinn ${ }^{\mathrm{a}}$ and Anne Rosser ${ }^{\mathrm{c}}$ \\ ${ }^{a}$ School of Health Care Studies, Cardiff University, Cardiff, UK \\ ${ }^{\mathrm{b}}$ Cardiff Brain Repair Group, School of Biosciences, Cardiff University, Cardiff, UK \\ ${ }^{\mathrm{c}}$ Institute of Psychological Medicine and Clinical Neurosciences, School of Medicine and \\ Cardiff Brain Repair Group, School of Biosciences, Cardiff University, Cardiff, UK
}

\begin{abstract}
Physical therapies and exercise may have potential as a disease modifying agent in Huntington's disease (HD) and in recent years, there have been several small scale feasibility studies that have shown benefit as a result of physical interventions. When evaluating complex physical interventions, a phased approach using mixed methodology designs that report specific intervention components, adherence, acceptability, adverse events and defined intervention protocols is important for replication and planning of future trials and to ensure potential for implementation in clinical practice.

A narrative review of the available literature related to physical activity, physical therapy and exercise in people with HD was performed using a population, intervention, comparison and outcome (PICO) approach. Eight studies met specific inclusion criteria and were reviewed in terms of their systematic conduct and reporting standards. All of the studies $(n=8)$ provided details of intervention including location and duration. The majority of interventions included balance training activities in combination with other complex activities of daily living that required therapist supervision. Two of the interventions were home based, the remainder were facility or hospital based. None of the studies reported adverse events whilst only $3 / 8$ reported adherence rates which were ranging from $60-80 \%$. In general, limited detail was provided on the specific individual components of the interventions.

This review of primary publications and conference proceedings, suggests that researchers working in the field need to focus on clearer reporting of intervention protocols so as to generate a better understanding of the impact of exercise and physical therapies on the symptoms of HD, as well as any potential synergistic role alongside the impending disease-modifying interventions.
\end{abstract}

Keywords: Huntington's disease, chorea, exercise, physical activity, physiotherapy, physical therapy, rehabilitation

\section{INTRODUCTION}

Huntington's Disease (HD) is an autosomal dominant neurodegenerative condition; the major features of the disease include cognitive deficits, behavioural changes and motor dysfunction. Although it is now well-documented that HD is a multimodal condition

*Correspondence to: Dr Monica Busse, School of Healthcare Studies, Ty DewiSant, Heath Park Site, Cardiff University, CF14 4XN, Cardiff, UK. Tel.: +44 029206 87559; E-mail: busseme@ cardiff.ac.uk.
$[1,2]$, we focus here on the motor disability. The most characteristic motor deficit is chorea, but it may be one of the least disabling elements of the motor phenotype; indeed with worsening functional capacity, chorea is reported to decrease whilst dystonia increases [3, 4]. Of the other many associated motor deficits, gait and balance disturbances are considered highly correlated to activity limitations in this population [5]. A high proportion of people with HD report frequent falling [5-7], and falls are a major factor implicated in admission to a nursing home [8]. Aetiology of falls in this population 
is likely to be multi-factorial [9], but observed deficits in gait and balance are considered the main contributing factors $[5,7]$.

To date there is no treatment that will prevent, delay or slow the progressive neurodegeneration, although there are ongoing international efforts to identify potential candidates [10-12]. A small number of treatments are available to ameliorate the chorea, with variable success, but no pharmacological treatments have been demonstrated to reduce the impact of most of the other movement deficits, including gait impairments and altered postural control.

A number of non-pharmacological approaches are also being explored in various international efforts. Deep brain stimulation (DBS) is being investigated in HD, following successes in Parkinson's disease (PD) [13], with individual case reports suggesting reduction of chorea and dystonia in the short term $[14,15]$. Other therapies such as primary-foetal neural-transplantation also have potential, although large scale clinical trials are still in the early stages of development $[16,17]$.

The likely role of combined genetic factors, other than the CAG repeat expansion, and as yet unidentified environmental influences on the progression of HD is generally accepted. The CAG repeat is suggested to account for up to $60 \%$ of the variance of age of onset $[18,19]$ but it is certain that other factors must contribute to the differences seen in progression rates for individuals with identical CAG expansion lengths [20-24]. These range from genetic modifiers to potential environmental toxins, to educational background and other life-style influences, including physical activity $[19,25]$. The potential for physical activities and therapies to influence symptom onset and progression in patients with $\mathrm{HD}$, or even to modify disease progression, has obvious important therapeutic implications both for its potential as a stand-alone intervention and as a moderator in ongoing therapeutic trials.

Environmental enrichment has been shown to retard the development of motor abnormalities in HD mouse lines with minimal amounts of enrichment associated with delayed loss of peristriatal cerebral volume. These studies [26-29] are frequently cited as providing evidence for the potential benefits for exercise in people with HD but importantly, environmental enrichment as defined in the literature typically encompasses three components, namely social enrichment, cognitive and physical activity. Indeed, a specific role for exercise as one component of the environmental enrichment effect has been difficult to convincingly demonstrate. Results from the handful of studies using the R6/1 HD mouse line have been disappointing [28, 30, 31]. Only modest recovery on secondary outcomes such as rearing or clasping behaviours, in the absence of improvements in primary outcomes such as general levels of activity or rotarod performance have been reported following running wheel exposure [30]. Although rotarod training (used as physical exercise) was found to be beneficial for subsequent rotarod performance in (female) R6/2 mice, it is difficult to determine whether this was truly an exercise benefit or a practice effect [28]. In another study, exercise was reported to have a negative effect of motor performance. It should also be noted that in this study, exercise in the transgenic mice did increase swimming speed in comparison to the non-exercised transgenic mice [31]. Consequently, it is at present unclear as to how beneficial exercise is to HD mice and a more refined and considered approach as to the nature, administration and experimental designs of the exercise studies in these mouse lines is required to fully understand the therapeutic value of exercise per se. It is unclear which modes of exercises should be considered in laboratory studies to be able to understand and differentiate causal relationships. Wheel running for example is a relatively simple motor task, and requires little problem solving and cognitive engagement. Participation in more difficult motor tasks that involve some skill acquisition, such as maze running, or reaching and grasping practice, may elicit greater brain activation and may result in improvement in both functional outcomes and delayed neural progression. Alternatively, implementation of exercises with a high aerobic content may be important for increasing cerebral blood flow, neurogenesis and related increases in growth factors such as BDNF, and may have beneficial effects on other aspects of function, such as cognition. What is required at present is a systematic comparison of different types of longer term exercise administration and cognitive training exposures directed at different neural substrates.

The design and implementation of exercise studies in animal models of HD has important implications for the consideration of exercise as a potential modifier to pharmacological or other interventions in both animal and human studies. Results from clinical studies, mostly time series and case reports [32,33] suggest that exercise may provide benefit to people with HD by minimising both impairments and improving function but a clear understanding of the nature of benefit eludes us, most likely due to the inadequate design and reporting of studies to date [32]. We are now at the stage at which it is important to conduct systematic controlled studies following the Medical Research 
Council (MRC) framework for complex interventions [34]. Furthermore, to guide translation of research into clinical practice, knowledge of detailed protocols, and retention, adherence and adverse events rates should be reported [35], especially as it cannot be assumed that exercise is necessarily safe in HD. For example, given the evidence for impaired mitochondrial function in muscle cells in HD, excessive exercise may be a potential concern [36]. To date however, there is only a single clinical case report suggesting such a detrimental effect, which was in a marathon runner with premanifest HD, who presented with myopathy years before the predicted disease onset [37].

Here we review the developing field of exercisebased physical interventions for people with HD and specifically discuss the relevant development of evidence in terms of safety and feasibility to support future clinical implementation. In order to gather robust evidence in support of exercise and physical activity as a therapeutic strategy, detailed components of the complex intervention as well as duration of intervention, level of supervision required, mode of delivery, location, equipment and costs should be well described. This will both help to distinguish between the different components of complex interventions which moderate outcome and to ensure wide spread and sustainable implementation of a specific intervention.

\section{Exercise in HD: A review of practice and progress}

In order to conduct this narrative review, a comprehensive literature search was conducted using AMED (Allied and Complementary Medicine), EMBASE <1947-Present>, Ovid MEDLINE(R) <1946 to May Week 1 2012>, PsycINFO <1806 to May Week 2 2012>, PsycArticles Full Text. Reference lists of systematic reviews were hand-searched. Key words were structured using a population, intervention, comparison and outcome (PICO) approach [38]. Population key words included 'Huntington', 'Huntington's disease', 'Huntington's chorea' and 'chorea'. Intervention keywords included 'physical therapy', 'physiotherapy', 'rehabilitation', 'exercise therapy', 'exercise', 'stretching', 'strengthening'. Outcomes were searched using the following key terms: 'daily living', 'activity' 'mobility', 'postural control', 'balance', 'posture', 'falls', 'function', 'gait', 'muscle strength', 'quality of life'. Studies were included if they investigated any physiotherapy or exercise-based intervention in people with Huntington's disease and reported the outcome with validated measures or with process evaluation. All experimental and quasi experimental trials were considered (conference proceedings and full text). Studies were excluded if all members of the population were aged $<18$ years and diagnosis of HD was not confirmed by a positive genetic test or a family history of HD with signs of chorea, if the intervention did not involve a physiotherapy or exercise component or did not report objective outcomes or was a process evaluation or a single case study. Articles meeting the review criteria were graded for study type and rated for quality using checklists to assess study validity and methodology. The level of evidence was classified as Level 1: interventions validated with RCTs with low false-positive (alpha) rates and high power; Level 2: intervention is supported by RCTs with high false-positive rates and low power; Level 3: nonrandomized comparisons between concurrent, matched groups; Level 4: nonrandomized group comparisons (including experimentally controlled single-case timeseries designs) and Level 5: case series without controls [39].

Eight studies met the inclusion criteria. The majority of studies that were excluded $[n=172$ retrieved; $n=164$ excluded by title or abstract] did not involve physiotherapy or exercise. Three studies were excluded as they were review papers related to the topic [32, 33,40 ] rather than primary research reports, and one study was excluded from the review as it did not provide detail of the intervention or outcomes [41]. Study design, participants, intervention type, outcomes and results are summarised in Table 1. More specific intervention details including location, supervision required, retention and adherence rates and any reports of adverse events are provided in Table 2 . Table 3 provides a summary of key points for critical appraisal using a format advocated by the Critical Appraisal Skills Programme (CASP).

One of the first studies that incorporated objective outcome measures was a time series study where the benefits of an inpatient rehabilitation programme including a specific physical therapy exercise programme were evaluated [42]. Whilst details of the specific content of each component of the rehabilitation programme was not provided, this study was an important landmark as it reported significant findings using well validated outcome measures, followed up patients over time, and consulted caregivers and family members about their opinions [43]. These researchers were both able to demonstrate benefit in objective outcomes as well as patient-perceived benefit using a mixed-method approach. They asked people with HD, who had completed at least one course of the 


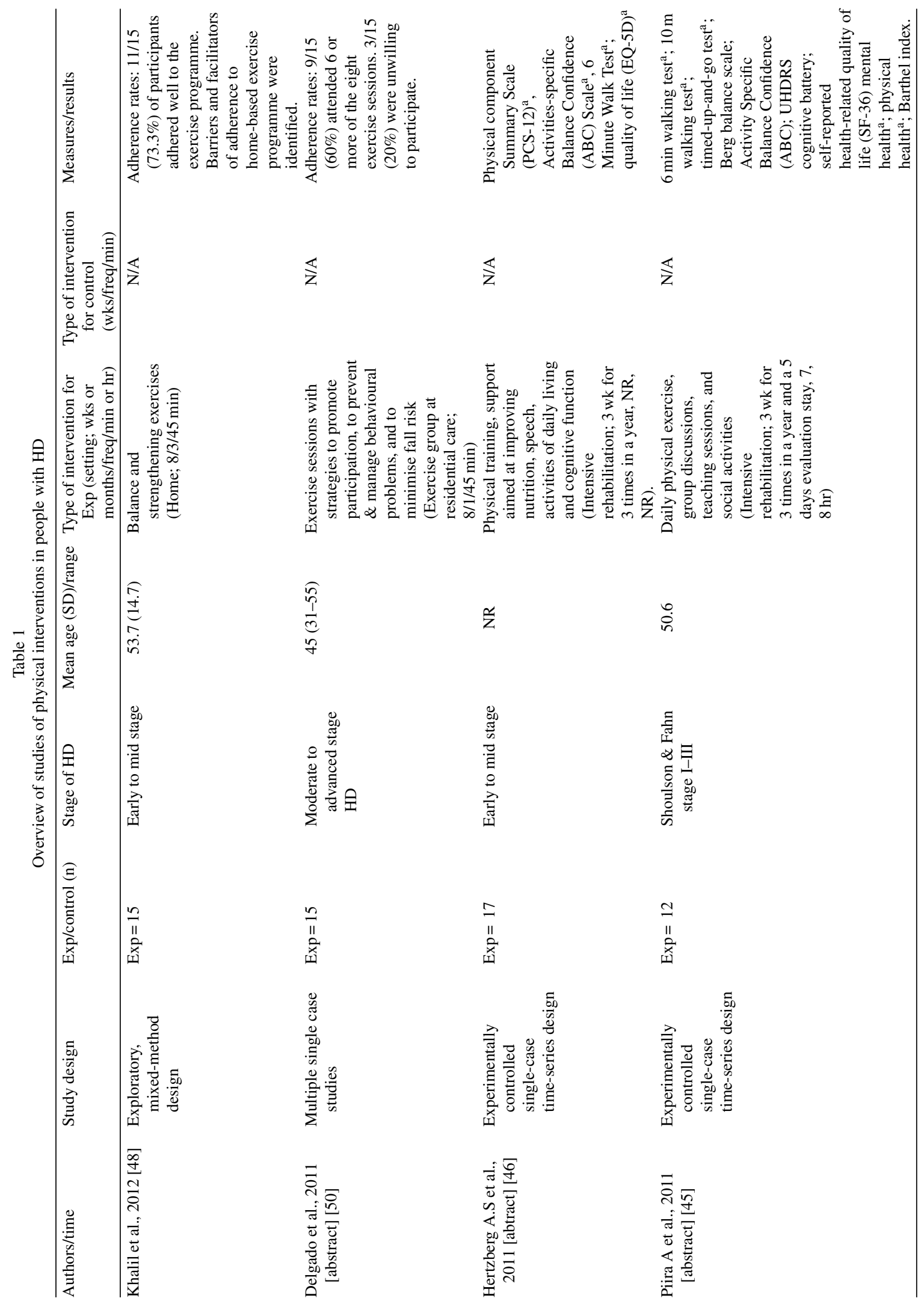




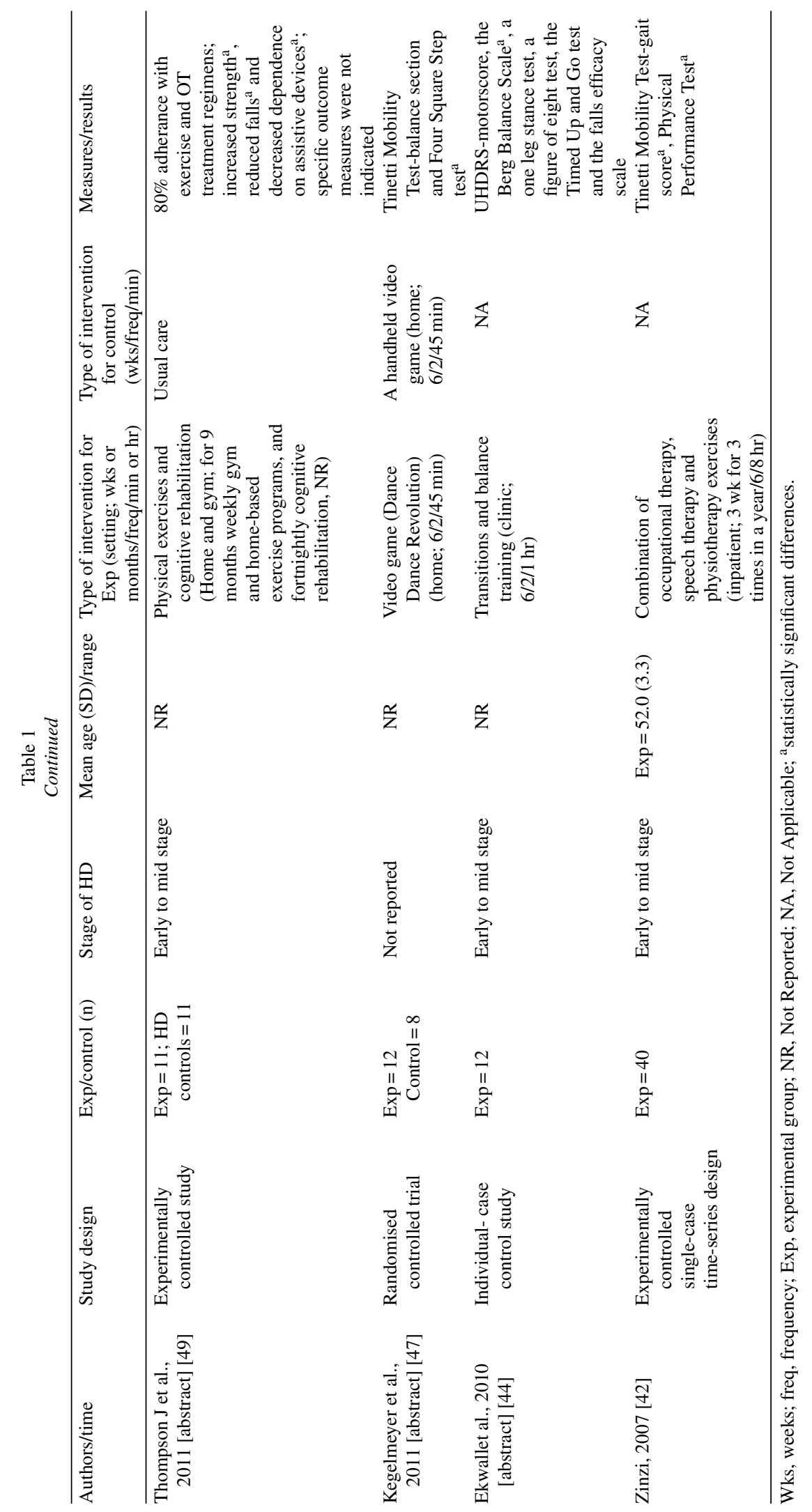




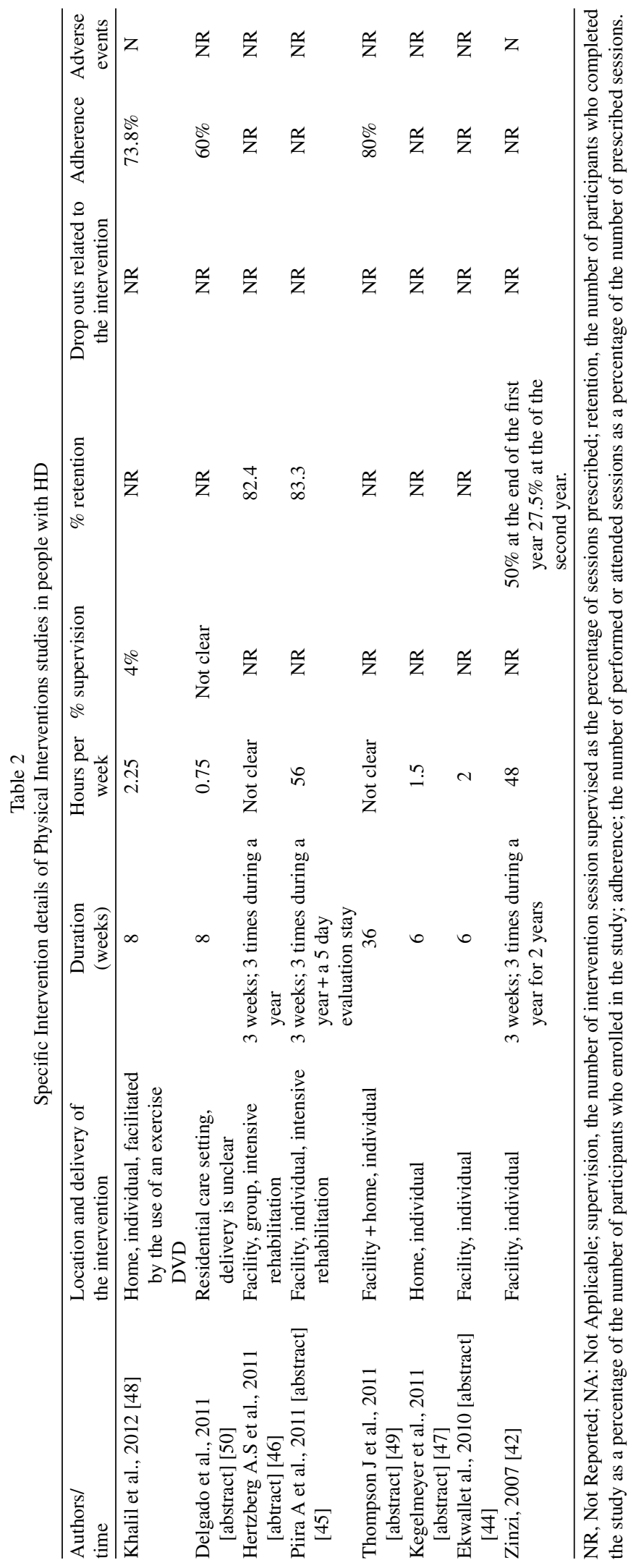




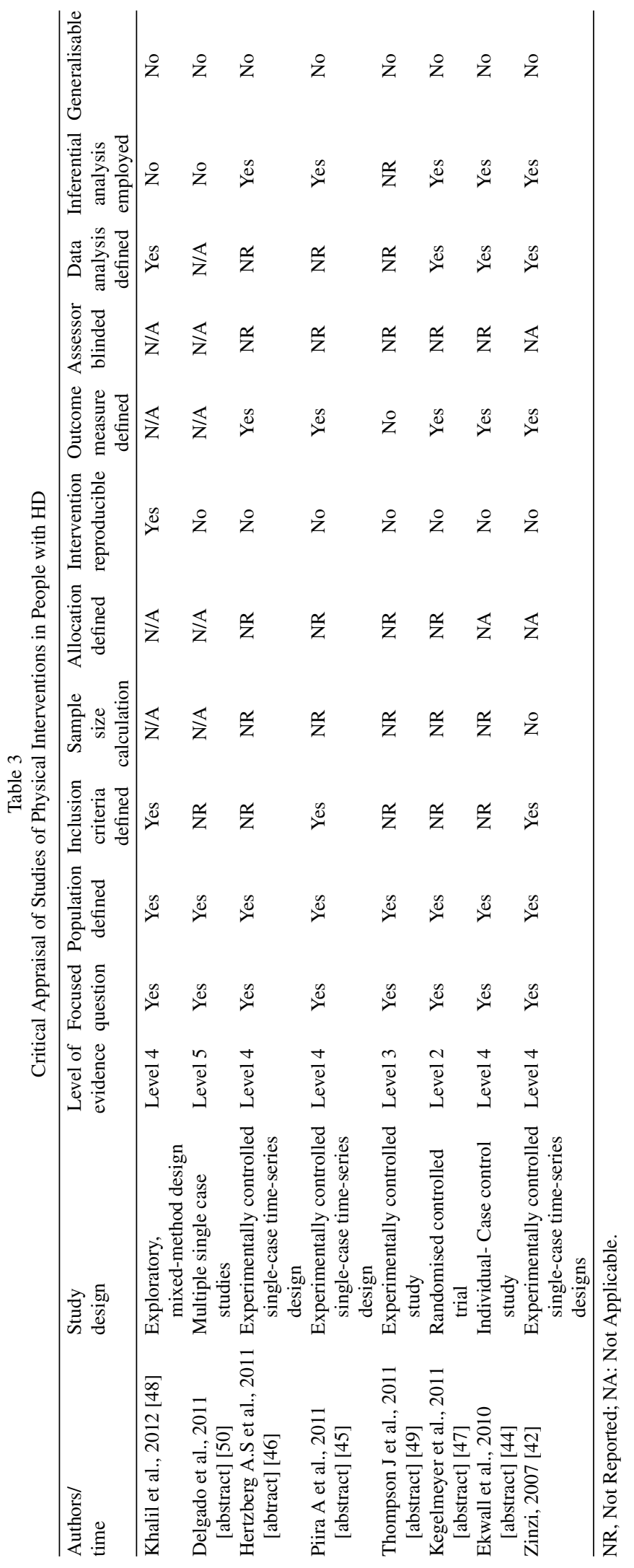


above-mentioned inpatient rehabilitation protocol, to evaluate their perspectives on their involvement in the rehabilitation programme. Nearly all the respondents $(n=37 / 40)$ perceived improvements after discharge. Improvements were reported in gait, balance and fall reduction. The duration of benefit was estimated to last between 1 and 3 months by $71 \%$ of the respondents. The majority of the respondents $(n=30 / 37$, $81 \%$ ) reported their intention to continue with the rehabilitation programme in the future, confirming the acceptability of the programme.

Since this first trial was reported in 2007, there has been a gradual increase in the reporting of feasibility and positive benefits from both in- and out-patient physiotherapy exercise programmes [44-46] as well as from home and gym based exercise programmes. There have been two reports (conference proceedings only) of replicative studies using similar protocols (both for the intervention and outcome assessment) $[45,46]$ to the first study by Zinzi et al. [42], both of which concluded that a multidisciplinary rehabilitation programme was associated with benefits in physical function and quality of life. Hertzberg et al. [46] additionally provided effect size data which would be useful in sample size calculations for larger scale clinical trials. The first reported controlled trial (conference proceedings only) of an exercise programme [47] to date was an exercise programme using a video game to improve dynamic balance and mobility in individuals with HD was evaluated in 20 subjects with early to mid-stage HD. Participants were randomly allocated to either the experimental $(n=12)$ or the control $(n=8)$ groups. The experimental group performed the video-based exercises for 45 minutes twice weekly for 6 weeks, whilst the control group performed a handheld video game. Those that adhered to the videobased exercise programme demonstrated significant improvement in a timed test of dynamic balance.

Three recent studies have focussed on feasibility and perceptions of exercise-based interventions in HD. Khalil et al. [48] conducted an exploration of how people with HD used a home-based exercise DVD and how it was perceived by the participants and their caregivers; adherence rates were high, however commitment of the caregiver was considered to be a key to the success of the programme. Thompson et al. (conference proceedings only) [49] assessed feasibility and protocol development for a tailored enrichment protocol encompassing physical exercises and cognitive rehabilitation delivered in the home environment over a 9-month period. The initial data suggested that it was feasible to deliver such an intervention and additionally that functional benefits were obtained in people with HD $(n=11)$ compared to non-intervention controls $(n=11)$ (no specific outcome measures were reported). High adherence to the intervention was documented. Delgado et al. (conference proceedings only) [50] aimed to investigate the feasibility of developing and running a group exercise programme, and to identify strategies to improve adherence and participation in an 8-week tailored exercise program for 15 mid-to late stage HD patients. Strategies to promote participation were specifically developed to manage behavioural problems, and consequently adherence rates were high, which is important given the stage of disease and the complex issues relating to behaviour and how that may impact upon exercise prescription. Adherence was reported however outcomes were not reported.

Physical interventions in HD are complex. The Medical Research Council (MRC) Framework for designing and evaluating complex interventions provides a methodology for evaluation of complex interventions utilising a cyclical development process whereby all the components are fully developed and evaluated in an iterative process so that an intervention is fully defined, developed and appropriate outcome measures considered before integration in large-scale clinical trials. There are a wide range of interacting components and behaviours that are required both by the persons receiving an exercise intervention (e.g. severity of the disease, medications, and individual personal factors) and by those delivering an physical intervention (e.g. information provision, prescribing different modes of exercise, and giving demonstrations) and it is important to be able to consider the influence of all of these components on outcomes [34]. An understanding of what the active ingredient of the complex intervention is and how it may be working is imperative so as to design more effective interventions that can be applied in a range of settings and relevant to specific impairments. A concrete example of this is the specific mode of exercise (i.e. aerobic versus non-aerobic and fitness based versus function focussed) that may have differential effects (central versus peripheral), which need to be appropriately considered. Clearly, the first step is that in order to effectively evaluate exercise as an intervention per se, research reports should provide appropriate detail not only of the intervention itself but also of the adherence, acceptability and any adverse events. All of the studies included in this review provided detail of location and duration of the intervention however the number of exercise hours per week was not reported in 2 of 
the 8 studies $[46,49]$ and the amount of supervision required was documented in only 2 of the 8 studies [42, 48]. Three of the 8 studies included reported exercise adherence rates [48-50], retention rates were provided in another 3 of the 8 studies $[42,45,46]$ and none of the studies provided reports of adverse events. The importance of publishing full trial data at this stage should be emphasised. The majority of studies reviewed here were conference proceedings; it is therefore difficult to establish whether the data is not available or simply not reported due to word limits in conference proceedings.

\section{Exercise in HD: Future directions}

Whilst exercise interventions have established efficacy in improving physical and cognitive function in individuals with various neurodegenerative diseases, including Alzheimer's Disease and PD [51-53], as well as Multiple Sclerosis [54], to date there have been no large scale clinical trials conducted in HD and most certainly no in-depth studies of the effect of exercise on brain function in HD.

In general, the small scale studies which have been conducted to date suggest that there is benefit in encouraging physical approaches and exercise in HD. We suggest that, whilst it is of utmost importance to strive for future large scale trials in support of the physical therapies, there is still work to do before reaching this point. Mixed methods designs that incorporate both quantitative and qualitative methodologies can be extremely useful in promoting an in-depth understanding and appropriate interpretation of the findings in studies of such complex interventions, particularly in the early stage studies that are being conducted in the HD field at this time. Managing the known barriers to physical activity $[55,56]$ and delivering physical interventions in practice requires a co-ordinated and creative approach where a variety of exercise options, implementation of behavioural strategies and careful planning is required [57] in order to ensure sustained uptake of specific targeted exercise interventions. Knowledge of acceptability, adherence, intervention delivery processes and recruitment and retention in small scale pilot studies is therefore important to be able to refine the design before moving to larger scale exploratory studies [34] that can ultimately be implemented into clinical practice.

This review, which includes a range of primary publications and conference proceedings, suggests that researchers working in the field need to focus on clear reporting of intervention protocols in proof of principle studies thus establishing feasibility in terms of acceptability, adherence and retention. Whilst the use of objective and sensitive functional outcome measures is certainly more apparent than in previous years in the studies reviewed here, an approach that focuses on understanding the active components and the interacting components and behaviours is still required. The choice of outcome measures is crucial to establishing benefit that is clearly linked to the underlying theoretical basis for the intervention itself and is important to aid mechanistic evaluations. End points for clinical trials [58] have been recommended for use in HD but few of these have been utilised in exercise studies to date. Additionally, the assessment of central effects of exercise eludes us and is an area for future research.

With better assessment and reporting, it will become possible to characterise both the impairment levels at which people with HD are able to get involved in regular exercise programmes and the types of support strategies that should be considered when delivering these kinds of interventions. It is also important to emphasise that although exercise may ultimately be a stand-alone intervention, it has a potentially synergistic role alongside the impending compounds and other therapeutic strategies, such as cell replacement therapy and forthcoming pharmacological interventions that the HD community are eagerly awaiting. Furthermore, given the potential of physical activity to influence function, consideration should be given to controlling and recording individuals' exercise levels (in particular any change in these levels) when investigating other potential therapeutic agents.

\section{ACKNOWLEDGMENTS INCLUDING SOURCES OF SUPPORT}

The authors have no conflicts of interest to declare. Ms Khalil has received research support from the Paul Jeffries Waters Bequest Fund to support research in Huntington's disease, the Overseas Research Studentship Fund and Jordan University of Science and Technology. Dr Quinn, Dr Busse and Professor Rosser have received research support from the Chief Medical Officer for Wales Research Budget, Wales Office for Research and Development, United Kingdom Huntington's Disease Association and the European Huntington's Disease Network (EHDN). Professor Rosser has research funding from the Medical Research Council and Paul Jeffries Waters Bequest. Dr Simon Brooks receives research support from Cure Huntingtons Disease Initiative (CHDI) and EHDN. 


\section{CONFLICT OF INTEREST}

None declared.

\section{REFERENCES}

[1] Naarding P, Kremer HP, Zitman FG. Huntington's disease: A review of the literature on prevalence and treatment of neuropsychiatric phenomena. Eur Psychiatry. 2001;16(8): 439-45.

[2] Craufurd D, Snowden J. Neuropsychological and neuropsychiatric aspects of Huntington's disease. Oxford Monographs on Medical Genetics. 2002;45:62-94.

[3] Feigin A, Kieburtz K, Bordwell K, Como P, Steinberg K, et al. Functional decline in Huntington's disease. Mov Disord. 1995;10(2):211-4.

[4] Marder K, Zhao H, Myers RH, Cudkowicz M, Kayson E, et al. Rate of functional decline in Huntington's disease. Huntington Study Group. Neurology. 2000;54(2):452-8.

[5] Grimbergen YAM, Knol MJ, Bloem BR, Kremer BPH, Roos RAC, et al. Falls and gait disturbances in Huntington's disease. Movement Disorders. 2008;23(7):970-6.

[6] Kloos AD, Kegelmeyer DA, Young GS, Kostyk SK. Fall risk assessment using the Tinetti mobility test in individuals with Huntington's disease. Movement Disorders. 2010;25(16):2838-44.

[7] Busse ME, Wiles CM, Rosser AE. Mobility and falls in people with Huntington's disease. Journal of Neurology, Neurosurgery \& Psychiatry. 2009;80(1):88-90.

[8] Wheelock VL, Tempkin T, Marder K, Nance M, Myers RH, et al. Predictors of nursing home placement in Huntington disease. Neurology. 2003;60(6):998-1001.

[9] Quinn L, Rao A. Physical therapy for people with Huntington disease: Current perspectives and case report. Journal of Neurologic Physical Therapy. 2002;26(3):145.

[10] Walker FO, Huntington's Disease. Semin Neurol. 2007;27(2):143-50.

[11] Ross CA, Tabrizi SJ. Huntington's disease: From molecular pathogenesis to clinical treatment. Lancet Neurol. 2011;10(1):83-98.

[12] Johnson CD, Davidson BL. Huntington's disease: Progress toward effective disease-modifying treatments and a cure. Hum Mol Genet. 2010;19(R1):R98-102.

[13] Hu W, Klassen BT, Stead M. Surgery for movement disorders. J Neurosurg Sci. 2011;55(4):305-17.

[14] Biolsi B, Cif L, Fertit HE, Robles SG, Coubes P. Long-term follow-up of Huntington disease treated by bilateral deep brain stimulation of the internal globus pallidus. J Neurosurg. 2008;109(1):130-2.

[15] Kang GA, Heath S, Rothlind J, Starr PA. Long-term follow-up of pallidal deep brain stimulation in two cases of Huntington's disease. J Neurol Neurosurg Psychiatry. 2011;82(3):272-7.

[16] Rosser AE, Dunnett SB. Neural transplantation in patients with Huntington's disease. CNS Drugs. 2003;17(12):853-67.

[17] Bachoud-Levi AC, Hantraye P, Peschanski M. Fetal neural grafts for Huntington's disease: A prospective view. Mov Disord. 2002;17(3):439-44.

[18] Penney JB, Vonsattel JP, MacDonald ME, Gusella JF, Myers RH. CAG repeat number governs the development rate of pathology in Huntington's disease. Ann Neurol. 1997;41(5):689-92.

[19] Gusella JF, MacDonald ME. Huntington's disease: The case for genetic modifiers. Genome Med. 2009;1(8):80.
[20] Panas M, Karadima G, Markianos M, Kalfakis N, Vassilopoulos D. Phenotypic discordance in a pair of monozygotic twins with Huntington's disease. Clin Genet. 2008;74(3):291-2.

[21] Friedman JH, Trieschmann ME, Myers RH, Fernandez HH Monozygotic twins discordant for Huntington disease after 7 years. Arch Neurol. 2005;62(6):995-7.

[22] Anca MH, Gazit E, Loewenthal R, Ostrovsky O, Frydman $\mathrm{M}$, et al. Different phenotypic expression in monozygotic twins with Huntington disease. Am J Med Genet A. 2004;124A(1):89-91.

[23] Georgiou N, Bradshaw JL, Chiu E, Tudor A, O'Gorman L, et al. Differential clinical and motor control function in a pair of monozygotic twins with Huntington's disease. Mov Disord. 1999;14(2):320-5.

[24] Wexler NS, Lorimer J, Porter J, Gomez F, Moskowitz C, et al. Venezuelan kindreds reveal that genetic and environmental factors modulate Huntington's disease age of onset. Proc Natl Acad Sci USA. 2004;101(10):3498-503.

[25] Trembath MK, Horton ZA, Tippett L, Hogg V, Collins VR, et al. A retrospective study of the impact of lifestyle on age at onset of Huntington disease. Mov Disord. 2010;25(10):144450 .

[26] Spires TL, Grote HE, Varshney NK, Cordery PM, Van Dellen A, et al. Environmental enrichment rescues protein deficits in a mouse model of Huntington's disease, indicating a possible disease mechanism. Journal of Neuroscience. 2004;24(9):2270-6.

[27] Hockly E, Cordery PM, Woodman B, Mahal A, Van Dellen A, et al. Environmental enrichment slows disease progression in R6/2 Huntington's disease mice. 2002;51(2):235-42.

[28] Wood NI, Carta V, Milde S, Skillings EA, McAllister CJ, et al. Responses to environmental enrichment differ with sex and genotype in a transgenic mouse model of Huntington's disease. PLoS One. 2010;5(2):e9077.

[29] Nithianantharajah J, Hannan AJ. Enriched environments: Experience-dependent plasticity and disorders of the nervous system. Nat Rev Neurosci. 2006;7(9):697-709.

[30] Pang TYC, Stam NC, Nithianantharajah J, Howard ML, Hannan AJ. Differential effects of voluntary physical exercise on behavioral and brain-derived neurotrophic factor expression deficits in huntington's disease transgenic mice. Neuroscience. 2006;(2):569-84.

[31] Potter M, Yuan C, Ottenritter C, Mughal M, van Praag H Exercise is not beneficial and may accelerate symptom onset in a mouse model of Huntington's disease. PLoS Currents. 2010;7(2):RRN1201.

[32] Bilney B, Morris ME, Perry A. Effectiveness of physiotherapy, occupational therapy, and speech pathology for people with Huntington's disease: A systematic review. Neurorehabilitation \& Neural Repair. 2003;17(1):12-24.

[33] Busse ME, Rosser AE. Can directed activity improve mobility in Huntington's disease? Brain Res Bull. 2007;72(2-3): 172-4.

[34] Craig P, Dieppe P, Macintyre S, Michie S, Nazareth I, et al Developing and evaluating complex interventions: The new Medical Research Council guidance. BMJ. 2008;337:a1655.

[35] Allen NE, Sherrington C, Suriyarachchi GD, Paul SS, Song J, et al. Exercise and motor training in people with Parkinson's disease: A systematic review of participant characteristics, intervention delivery, retention rates, adherence, and adverse events in clinical trials. Parkinsons Dis. 2012;2012:854328.

[36] Saft C, Zange J, Andrich J, Muller K, Lindenberg K, et al. Mitochondrial impairment in patients and asymptomatic mutation carriers of Huntington's disease. Movement Disorders. $2005 ; 20(6): 674-9$. 
[37] Kosinski CM, Schlangen C, Gellerich FN, Gizatullina Z, Deschauer M, et al. Myopathy as a first symptom of Huntington's disease in a Marathon runner. Movement Disorders. 2007;22(11):1637-40.

[38] Higgins JPT, Sally Green P. Cochrane handbook for systematic reviews of interventions. John Wiley \& Sons; Vol. 4. 2011.

[39] Sackett DL. Rules of evidence and clinical recommendations on the use of antithrombotic agents. Chest. 1989;95 (2 Suppl)2S.

[40] Giladi N. Mobility and exercise in movement disorders. Parkinsonism Relat Disord. 2009;15(Suppl 3)S46-8.

[41] Vieira R, Inacio D, LP, d.S.F, L, C. Physical therapy in Huntington's disease: Report of cases. Revista Neurociencias. 2011;19(3):504-11.

[42] Zinzi P, Salmaso D, De Grandis R, Graziani G, Maceroni $\mathrm{S}$, et al. Effects of an intensive rehabilitation programme on patients with Huntington's disease: A pilot study. Clinical Rehabilitation. 2007;21(7):603-13.

[43] Zinzi PSD, Frontali M, Jacopini G. Patients' and caregivers' perspectives: Assessing an intensive rehabilitation programme and outcomes in Huntington's disease Journal of Public Health. 2009;17(5):331-8.

[44] Ekwall C, Bohlen S, Reilmann R, Wiklund L. J02 Physical therapy for patients with Huntington's disease: Effects of a treatment program and intercorrelation between outcome measures. Journal of Neurology, Neurosurgery \& Psychiatry. 2010;81(Suppl 1):A40.

[45] Piira A, Oie L, Nilsen T, Knutsen S, Van Walsem M, et al. A multidisciplinary intensive rehabilitation program for persons with Huntington's disease - Preliminary results after 1 year. In World Congress on Huntington's Disease. Melbourne, VIC Australia: Clinical Genetics; 2011.

[46] Hertzberg A, Lindeman A, Haavik-Nilsen K, Frich J. Results from an intensive rehabilitation programme for persons with Huntington's disease. In World Congress on Huntington's Disease. Melbourne, VIC Australia: Clinical Genetics; 2011.

[47] Kegelmeyer D, Fritz N, Kostyk S, Kloos A. J04 The effect of video game-based exercise on dynamic balance and mobility in individuals with Huntington's disease. Journal of Neurology, Neurosurgery \& Psychiatry. 2010;81(Suppl 1):A40-A40.

[48] Khalil H, Quinn L, van Deursen R, Martin R, Rosser A, et al. Adherence to Use of a Home-Based Exercise DVD in
People With Huntington Disease: Participants' Perspectives. Physical Therapy. 2012;12(1):69-82.

[49] Thompson J, Cruickshank T, Penailillo L, Carey-Hill W, Davies S, et al. The effects of environmental enrichment in a human Huntington's disease population. In World Congress on Huntington's Disease. Melbourne, VIC Australia: Clinical Genetics; 2011.

[50] Delgado V, Lownie A, Loy C. Achieving group exercise program participation and adherence, among people with moderate to advanced HD in care. In World Congress on Huntington's Disease. Melbourne, VIC Australia: Clinical Genetics; 2011.

[51] Cotman CW, Berchtold NC. Exercise: A behavioral intervention to enhance brain health and plasticity. Trends Neurosci. 2002;25(6):295-301.

[52] Deslandes A, Moraes H, Ferreira C, Veiga H, Silveira H, et al. Exercise and mental health: Many reasons to move. Neuropsychobiology. 2009;59(4):191-8.

[53] Goodwin VA, Richards SH, Taylor RS, Taylor AH, Campbell JL. The effectiveness of exercise interventions for people with Parkinson's disease: A systematic review and meta-analysis. Mov Disord. 2008;23(5):631-40.

[54] Rietberg M, Brooks D, Uitdehaag B, Kwakkel G. Exercise therapy for multiple sclerosis. Cochrane Database Syst Rev. 2005;1:1-26.

[55] Elsworth C, Dawes H, Sackley C, Soundy A, Howells K, et al. A study of perceived facilitators to physical activity in neurological conditions. International Journal of Therapy and Rehabilitation. 2009;16:17-23.

[56] Quinn L, Busse M, Khalil H, Richardson S, Rosser A, et al. Client and therapist views on exercise programmes for early-mid stage Parkinson's disease and Huntington's disease. Disability and Rehabilitation. 2010;32(11):917-28.

[57] Gambiza M. Overcoming barriers to physiotherapy provision for people with Huntington's disease in a residential setting. In World Congress on Huntington's Disease. Melbourne, VIC Australia: Clinical Genetics; 2011.

[58] Tabrizi SJ, Reilmann R, Roos RA, Durr A, Leavitt B, et al. Potential endpoints for clinical trials in premanifest and early Huntington's disease in the TRACK-HD study: Analysis of 24 month observational data. Lancet Neurology. 2012;11(1): 42-53. 